\title{
Telemedical Transport Layer Security based Platform for Cardiac Arrhythmia Classification using Quadratic Time-Frequency Analysis of HRV Signal
}

\author{
Ismail HADJ AHMED ${ }^{1}$ • Abdelghani DJEBBARI ${ }^{1}$ • Amar KACHENOURA ${ }^{2}$ • Lotfi \\ SENHADJI $^{2}$
}

Received: date / Accepted: date

\begin{abstract}
In this paper, we have developed a client-server model implemented as a telemedical platform for real-time remote monitoring of the cardiovascular function in patients suffering from arrhythmia. This technical platform capable of detecting and classifying in real-time cardiac arrhythmia via a Graphical User Interface (GUI) using Time-Frequency analysis methods, features extraction, features selection, and classification of the Heart Rate Variability signal recorded using a data acquisition system. The data acquisition system that we used is mainly designed around the Raspberry Pi zero which communicates with a server through the TCP/IP involving a $4 \mathrm{G} / 3 \mathrm{G}$ connection secured by the transport layer security (TLS) for a reliably safe connection between client and server. This telemedical platform adopts continuous control and monitoring of the heart rhythm. By using this system, in case of an alarm, medical staff can easily communicate with their patients in the hospital or at home.
\end{abstract}

Keywords Heart rate variability, Smoothed pseudo Wigner-Ville Distribution, Support Vector Machine, Mutual Information, Feature Selection with Adaptive Structure Learning, Transport Layer Security.

\section{Nomenclature}

ADC Analog-Digital Converter

Ismail HADJ AHMED , Abdelghani DJEBBARI

E-mail: $\{$ ismail.hadjahmed, abdelghani.djebbari\} @univ-tlemcen.dz . Amar KACHENOURA, L. SENHADJI

E-mail:\{ amar.kachenoura, Lotfi SENHADJI \}@univ-rennes1.fr

${ }^{1}$ Laboratory of Biomedical Engineering, Department of Biomedical Engineering, Faculty of technology, University of Tlemcen, Tlemcen, Algeria

${ }^{2}$ University of Rennes, Inserm, LTSI - UMR 1099, F-35000 Rennes, France
CPU Central Processing Unit

ECG Electrocardiogram

FS Features selected

FSASL Feature Selection with Adaptive Structure Learning, Transport Layer Security

FSM Features selection methods

HRV Heart Rate Variability

IoT Internet of Things

IP Internet Protocol

MI Mutual Information

NF Number of features

SPI Serial Peripheral Interface

SVM Support Vector Machine

TCP Transmission Control Protocol

TF Time-Frequency

TLS Transport Layer Security

\section{Introduction}

Cardiovascular diseases are a major cause of a very large number of chronic illness and disability around the world. Every year, millions of people die due to cardiac arrhythmias. Cardiac arrhythmias are due to alterations in the heart's electrical system that prevents it to beat properly. It causes the heart to beat too fast (tachycardia), too slow (bradycardia), or in any other irregular pattern. Some types of arrhythmia are harmless, but they can induce annoying symptoms like dizziness, and fainting. Other types of arrhythmia are more dangerous and can lead to serious complications, such as: cardiac sudden death, stroke, heart failure, etc. The beatto-beat duration variation, known as the Heart Rate Variability (HRV) signal, is modulated by the sympathetic and the parasympathetic activities of the Autonomic Nervous System (ANS) [1, 2]. The HRV signal can be generated by $\mathrm{R}$-peak detection in the Electrocardiogram (ECG) signals. 
Several studies, based on the analysis of HRV signals, offer a diagnosis aid for arrhythmia detection and characterization. More particularly, time, frequency, time-scale, and time-frequency domain were investigated in several studies for extraction of some specific features [3-7]. Classification techniques, such as Support Vector Machine (SVM), Artificial Neural Networks (ANN) and decision trees, were used to analyze HRV signals for diagnosis aid purposes [8-12]. These classifiers are applied directly on HRV signals or on features of interest extracted from this signal on different domains.

Within the context of the Internet of Things, some research group developed applications for the Health monitoring system of the cardiovascular function (see Table 5). Danan Thilakanathanet al. [13] have built a remote caring system for patients at home using ECG sensors, cloud storage providers, and mobile technology. This work consists of finding solutions that allow patients to share their health information with the medical professional team in a reliably secure and confidential manner. M. Shamim Hossain et al. [14] have used IoT technologies for ECG health monitoring, where ECG signals have been recorded via ECG sensors at home and sent the data to smartphones or computers via Internet. Smartphones were used as a client side that allows processing the ECG signal (removes unwanted noise from the recorded signal) and detecting the R-peaks. The acquired ECG signal was then transmitted to a server for analysis and characterization; the temporal and spectral characteristics were extracted and classified using a SVM classifier. Their classification results can be sent to the medical professional team for the analysis and diagnosis, where they transfer the medical report later to the cloud server for the patient to be informed. R. Lakshmi Devi et al. [15] have developed a classification system of cardiac arrhythmia using IoT that enables the ECG monitoring system to analyze the signal acquired from the data acquisition system (AD8232 \& Arduino). The statistical features were extracted from the HRV signals in the temporal domain, and used as inputs for a SVM classifier to identify the cardiac arrhythmia disease. The acquired ECG signal, processing, and classification tasks were done inside the system, which sends the result to a smartphone. Khalid abusalim et al. [16] have used an IoT ECG monitoring system that allows sending the ECG data to a cloud server via wireless networks with a security system using share the health information system (A system of encryption and decryption algorithm). The system was developed based on the storage, processing, and classification of cardiac arrhythmia from different healthy subjects available in the MIT-BIH. This study used three different types of features that were extracted from the ECG signals: time-domain, frequency-domain, and timefrequency plane. $\mathrm{K}$-nearest neighbor is used as a classification approach in this study to identify and classify the cardiac arrhythmia. In this paper, we developed a clientserver model implemented as a telemedical platform for real time-remote monitoring of the cardiovascular function in arrhythmia patients. This system allows continuous recording when the ECG Sensor is attached to the patient's body. The ECG is firstly acquired using data acquisition system designed around the Raspberry Pi zero which communicates with a server through the TCP/IP secured by the Transport Layer Security in order to secure the exchange of ECG data transmitting over the internet network. A HRV times series is then extracted from the acquired ECG signals and analyzed by Quadratic time-frequency distributions, to calculate new $\mathrm{TF}$-features to differentiate between various cardiac pathologies such as Atrial Fibrillation, SupraVentricular Tachycardia, and Congestive Heart Failure by analyzing their respective HRV signals. More precisely, the TFfeatures of interest are selected by means of the Mutual Information and the Feature Selection with Adaptive Structure Learning. These features are used as inputs for a SVM classifier to identify the acquired ECG among the different classes of the implemented training database. The system alerts the medical professional when the patient is detected as affected by cardiac arrhythmia disease according to the result of classification. The professional then sends the medical report to the cloud server and the cloud then warns the patient. Indeed our client-server architecture allows the cardiologist to monitor multiple patients at home or in hospital in parallel and in the same time.

\section{Materials and Methods}

Our system is composed of two main blocs (see figure 1): a hardware block, named the client block, devoted to the acquisition and the transmission of the ECG, and a software block, called server block, dedicated to HRV extraction, analysis and classification (features extraction, features selection, and patient classification)(see figure $8(\mathrm{~b})$ ). The client block consists mainly of (1) the analog shaping circuit ECG AD8232, (2) the analog-to-digital converter MCP3008, (3) the data acquisition card (Raspberry Pi zero) to acquire ECG signal. The sampling frequency is ensured by the internal analog-to-digital (ADC) converter of the MCP3008 it has been setup at $100 \mathrm{~Hz}$. Regarding the server block, the Physionet Bank [17] is used to choose the best features and the SVM parameters that provides the high classification rate between 2 classes of cardiac arrhythmias, namely AF and SVT among NSR and CHF. More precisely, our approach is based on a high-resolution TF analysis of the HRV signal that provides features as input for the SVM classifier. To do so, high-resolution quadratic time-frequency analysis methods are used for detecting the non-stationary content of the HRV signals. Indeed, Quadratic Time-Frequency Distributions (QTFDs) are widely known for their separable 


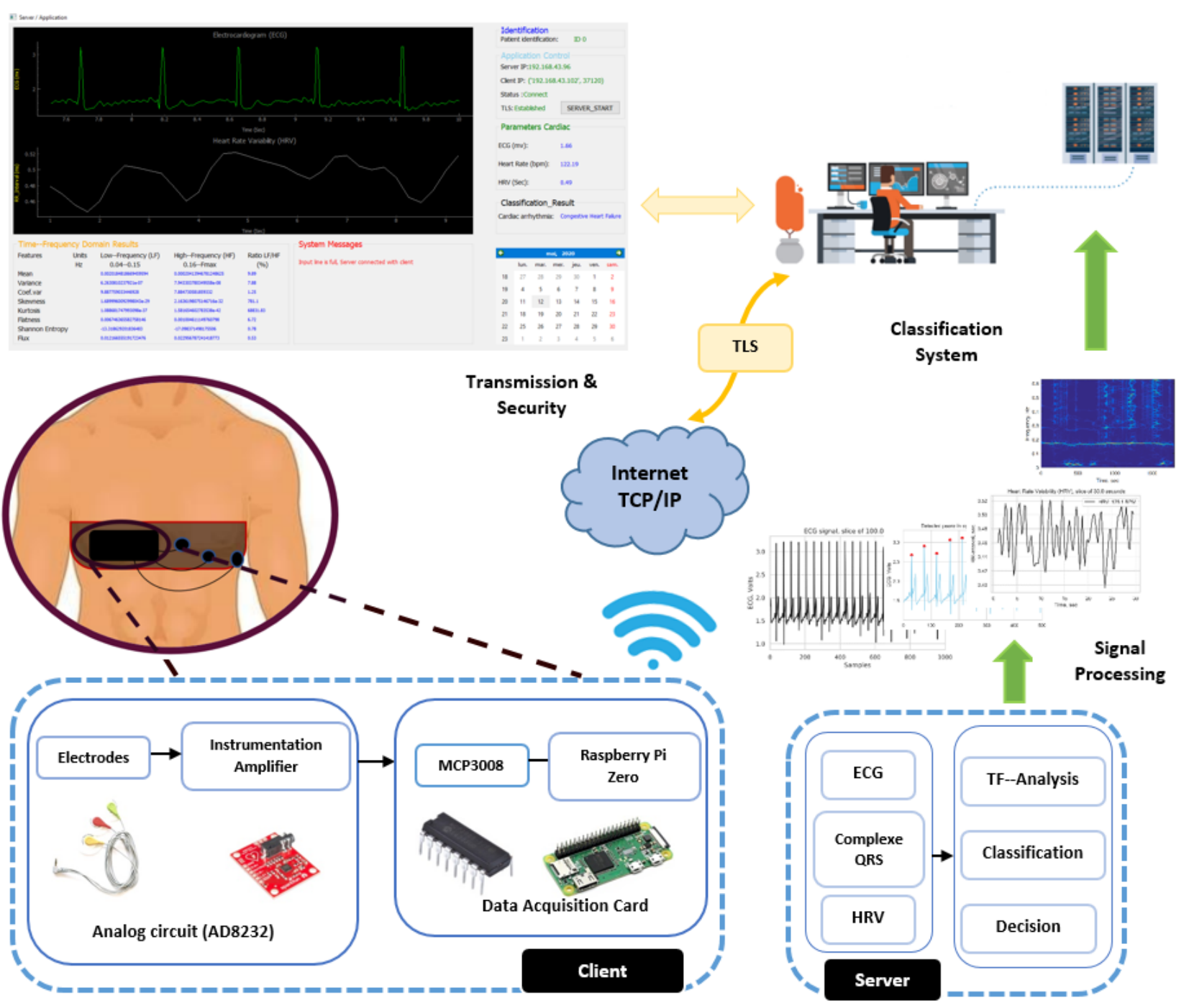

Fig. 1: Block diagram of a Telemedical platform for monitoring and classification of cardiac arrhythmias in real-time.

kernels which ensure better characterization of the transient behavior of HRV signals. The server block functions can be divided into four main subblocks. First, a QTFDs of HRV signals is calculated. Second, a set of 8 features are extracted from the TF-representation of HRV signal. Thirdly, a features selection technique is carried out using two different methods: FSASL algorithm, and MI technique to provide convenient features that yield the best classification performance. Finally, machine learning is carried out through the SVM classifier. Note that, a HRV signal of a new subject is then classified using all the parameters learned before, namely the best features and the SVM parameters. Graphical User Interface (GUI) developed within a Python environment is also provided (figure 1).

\subsection{Dataset}

In order to select the best features and the parameter of the SVM classifier, the normal and abnormal subjects were collected from the PhysioNet research repository [17]: congestive heart failure RR interval database (chf2db), BIDMC congestive heart failure database (chfdb), MIT-BIH Arrhythmia database (MITdB), LongTerm AF database (LTAFdB), Supraventricular tachycardia (SVDB), St. Petersburg Institute of Cardiological Technics 12-lead Arrhythmia (INCARTdB), and MIT-BIH normal sinus rhythm database (NSRDB). A total of 44 NSR, 77 SVT, $63 \mathrm{AF}$, and 44 CHF ECG are exploited in our study. Collected data, shown in Table 1 , are formed by 228 signals which we reorganized into four classes namely: SVT, NSR, AF, and CHF. 
Table 1: ECG Database collected from Physionet.

\begin{tabular}{|c|c|c|c|c|}
\hline \multirow{2}{*}{ Classes } & \multicolumn{4}{|c|}{ Data base details } \\
\hline & Data base & Number of ECG & Sampling Frequency $(\mathrm{Hz})$ & Duration (h/min) \\
\hline \multirow{3}{*}{ Atrial Fibrillation (AF) } & INCARTdB & 4 & 257 & $30 \mathrm{~min}$ \\
\hline & MITdB & 6 & 360 & $30 \mathrm{~min}$ \\
\hline & LTAFdB & 53 & 128 & $24 \mathrm{~h}$ \\
\hline \multirow{3}{*}{ Normal sinus rhythm (NSR) } & INCARTdB & 3 & 257 & $30 \mathrm{~min}$ \\
\hline & MITdB & 23 & 360 & $30 \mathrm{~min}$ \\
\hline & NSRDB & 18 & 128 & $24 \mathrm{~h}$ \\
\hline \multirow[t]{2}{*}{ Supraventricular tachycardia (SVT) } & SVDB & 77 & 128 & $30 \mathrm{~min}$ \\
\hline & $\operatorname{chf} 2 \mathrm{db}$ & 29 & 128 & $24 \mathrm{~h}$ \\
\hline Congestive Heart Failure (CHF) & $\operatorname{chfdb}$ & 15 & 250 & $20 \mathrm{~h}$ \\
\hline
\end{tabular}

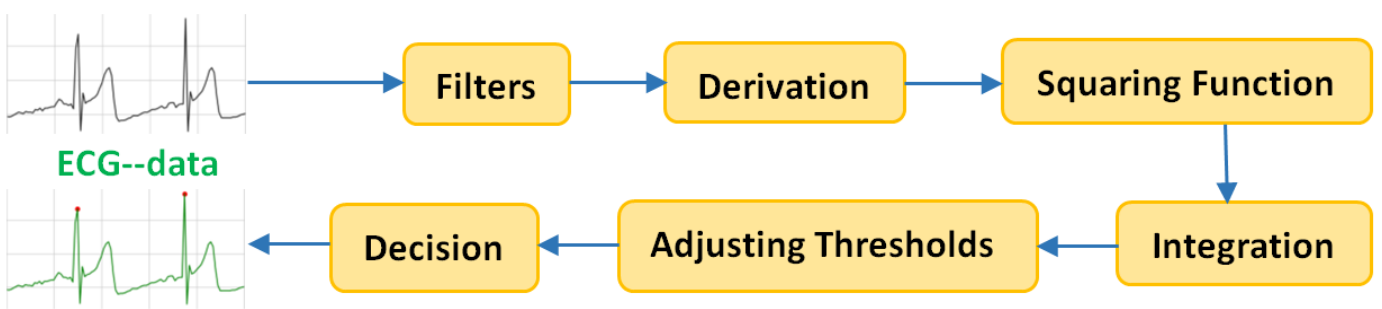

R--Peaks

Fig. 2: QRS detector based on the Pan-Tomkins algorithm.

\subsection{Detection of Heart Rate Variability (HRV)}

We generated HRV signals by detecting QRS-complexes within Electrocardiogram (ECG) signals by means of the Pan-Tompkins algorithm [18]. Pan-Tompkins algorithm can be summarized in figure 2 as follow:

The ECG-data is firstly filtered using a band pass to remove baseline and noise with high frequency. Then the filtered ECG signal is derived to highlight the slope of the R wave. Then, the signal is squared and a moving average filter is applied to provide information about the slope of $\mathrm{R}$ wave. Two thresholds are automatically adjusted to float over the noise.

\subsection{Quadratic Time-Frequency Analysis}

TF representation helps overcoming limitations of both temporal and spectral analysis methods of non-stationary signals such as HRV signals. More specifically, QTFDs allow to represent the energy of a signal over both time and frequency domains at a high joint time-frequency resolution [19]. QTFDs mainly satisfy time-shift and frequency-shift in variance properties. This allows QTFDs to provide appropriate representations of multicomponent non-stationary signals. Formally, QTFDs can be expressed as given in (1) $[19,20]$;

$\rho_{z}(t, f)=\int_{-\infty}^{+\infty} A F(\nu, \tau) g(\nu, \tau) e^{j 2 \pi(t \nu-f \tau)} \mathrm{d} \nu \mathrm{d} \tau$

where $g(\nu, \tau)$ is the smoothing kernel defined in the Dopplerlag domain as given in (2);

$g(\nu, \tau)=G_{1}(\nu) g_{2}(\tau)$

where $G_{1}(\nu)$ is the Doppler window, and $g_{2}(\tau)$ is the lag window. The Ambiguity Function (AF) is defined as given in (3);

$A F(\mu, \tau)=\int_{-\infty}^{+\infty} R(t, \tau) e^{-j 2 \pi \nu t} \mathrm{~d} t$

where $R(t, \tau)$ represents the time-dependent instantaneous auto-correlation of $z(t)$, the analytic version of the analyzed signal $s(t)$, as given in (4);

$R(t, \tau)=z\left(t+\frac{\tau}{2}\right) z^{*}\left(t-\frac{\tau}{2}\right)$ 
Because of its quadratic nature, the classical QTFDs methods may suffer from cross-terms that can considerably blur the TF plane. These interferences should be alleviated by adding filters over time and frequency domains in the quadratic distribution.

\subsubsection{Smoothed Pseudo Wigner-Ville Distribution $(S P W V D)$}

According to (4), the quadratic nature of smoothed versions of the Wigner-Ville distributions inevitably causes crossterms occurrence within the TF plane. Therefore, smoothing the TF plane over time and frequency domains is necessary to enhance auto-terms representation within the TF plane. A high resolution in time and frequency can be achieved by using separable time and frequency smoothing kernel. This yield to the Smoothed Pseudo Wigner-Ville Distribution (SPWVD), which is defined by (5);

$$
\begin{aligned}
& S P W V D(t, f)= \\
& \int_{-\infty}^{+\infty} g_{2}(\tau) \int_{-\infty}^{+\infty} g_{1}(s-t) z\left(s+\frac{\tau}{2}\right) z^{*}\left(s-\frac{\tau}{2}\right) \mathrm{s} e^{-2 \pi \nu \tau} \mathrm{d} \tau
\end{aligned}
$$

Smoothing carried out by the SPWVD is achieved at the price of spread auto-terms bursts within the TF plane. Therefore, smoothing windows have to be set to keep a representative TF distribution of the analyzed signal.

\subsection{Feature extraction}

In this study, we are interested in typical frequency bands that are usually explored during spectral analysis of HRV signals; namely, the LF and the HF band. We investigated these bands by computing their respective cumulative features such as: flux, flatness, Shannon entropy, mean, variance, skewness, kurtosis, and the coefficient of variation. In order to quantify each frequency band within the overall energy of the analyzed HRV signal, we calculate energy-based features of the HRV signal over LF and HF bands within the TF plane (contrary to the most existing studies which just exploit the frequency domain). More precisely, for both LF and HF bands, eight features are extracted from the TF plane of the analyzed HRV signals i) three TF energy-based features, and ii) five TF statistical-based features.

\subsubsection{TF energy-based features}

Flux The spectral flux is an estimation of the spectral change of the analyzed signal. It is calculated by measuring and comparing the difference between squared magnitudes of the Fourier transform of two adjacent frames of the power spectrum. The spectral flux can be used to estimate the distribution energy content in the both time and frequency domains by estimating changes of the TF distribution, as given in (6) [21];

$$
F L_{(t, f)}=\sum_{n=1}^{N-l} \sum_{k=1}^{M-m}\left|\rho_{z}[n+l, k+m]-\rho_{z}[n, k]\right|
$$

where $\rho_{z}[n, k]$ represents the TF representation of the analyzed HRV signal (size NxM) of the analytic version $z[n]$ of the analyzed signal $s[n]$, and $l$ represents the time duration between the two slices. The spectral flux is used to measure the change behavior of the power spectrum of a signal.

Flatness The spectral flatness is defined as the ratio between the geometric mean and the arithmetic mean of a power spectrum of a signal within the time domain. The spectral flatness of TF representations of size NxM is calculated by measuring the ratio between the geometric mean and the arithmetic mean of the energy distribution over the TF plane, as given in (7) [21, 22];

$S F_{(t, f)}=M N \frac{\prod_{n=1}^{N} \prod_{k=1}^{M}\left|\rho_{z}[n, k]\right| \frac{1}{N M}}{\sum_{n=1}^{N} \sum_{k=1}^{M} \rho_{z}[n, k]}$

Normalized Shannon Entropy The normalized Shannon entropy is a concentration measure which can be extended to a TF representation as given in (8);

$$
S E_{(t, f)}=-\sum_{n=1}^{N} \sum_{k=1}^{M} \frac{\rho_{z}[n, k]}{\sum_{n} \sum_{k} \rho_{z}[n, k]} \log _{2} \frac{\rho_{z}[n, k]}{\sum_{n} \sum_{k} \rho_{z}[n, k]}
$$

The Shannon entropy which is an extension of spectral entropy is a limit case of the Renyi entropy.

\section{Statistical-based features}

Mean The mean value calculated for the TF distribution [19] is given by (9);

$m_{(t, f)}=\frac{1}{N M} \sum_{n} \sum_{k} \rho_{z}(n, k)$

Variance The TF variance, which represents the spread of the TF distribution [19], is given as follow (10);

$$
\sigma_{(t, f)}^{2}=\frac{1}{N M} \sum_{n} \sum_{k}\left(\rho_{z}(n, k)-m_{(t, f)}\right)^{2}
$$


Skewness The skewness represents the asymmetry of the probability distribution of the energy from its mean. This parameter can be defined in TF plane [19] by (11);

$\gamma_{(t, f)}=\frac{1}{N M \sigma_{(t, f)}^{3}} \sum_{n} \sum_{k}\left(\rho_{z}[n, k]-m_{(t, f)}\right)^{3}$

Kurtosis The kurtosis measures whether the data are heavytailed or light-tailed relative to a normal distribution. In the TF plane [19], it is given by the following equation (12);

$k_{(t, f)}=\frac{1}{N M \sigma_{(t, f)}^{4}} \sum_{n} \sum_{k}\left(\rho_{z}[n, k]-m_{(t, f)}\right)^{4}$

Coefficient of variation The coefficient of variation is defined as the ratio between the variance and the mean in the TF plane [19], and is given by (13);

$c_{(t, f)}=\frac{\sigma_{(t, f)}}{m_{(t, f)}}$

\subsection{Feature selection}

Several feature selection techniques exist that optimize classification. Feature selection can be achieved through filter methods, wrapper methods, and embedded methods [23]. Filter methods are based on ranking features to select highly ranked features to be used as inputs for classifier. Wrapper methods are based on searching for the best feature subsets to get high-performance metrics of the predictor. Embedded methods focus on the training process without segmentation of data into training and test data to select relevant features [24]. In this study, we use two efficient methods: MI algorithm and FSASL method $[25,26]$.

\section{Mutual information (MI)}

We used the MI as a feature selection criterion. The aim of this approach is to distinguish relevant features within a particular class in order to create a subset formed by highly ranked features. The mutual information between two random variables $X$ and $Y$ is a measure of their mutual dependence [25], and is defined as given in (14);

$I(X, Y)=\sum_{x, y} p_{X, Y}(x, y) \log \frac{p_{X, Y}(x, y)}{p_{X}(x) p_{Y}(y)}$

where $p_{X}$ and $p_{Y}$ represent probability density functions (pdf) of $\mathrm{X}$ and $\mathrm{Y}$, respectively, and $p_{X, Y}$ is the joint pdf of $\mathrm{X}$ and $\mathrm{Y}$. The mutual information between features $V=$ $(v 1, v 2, \ldots, v d)$ and class variables $C=\left(c_{1}, c_{2}, \ldots, c_{k}\right)$ is expressed as given in (15);

$I(V, C)=\sum_{c} p(v) \sum_{c} p(v / c) \log \frac{p(v / c)}{p(v)}$
The MI is based on the measure of dependency between the variables to reduce number of features. This type of feature selection methods is symmetric and nonnegative. The MI can be equal to zero when the variables are independent.

Feature Selection with Adaptive Structure Learning (FSASL)

Unsupervised feature selection methods choose the features that can reveal or maintain the underlying structure of data [26]. The feature Selection with Adaptive Structure Learning (FSASL) considered a wrapper approach; it's based on linear regression. The formulation of FSASL is:

$$
\begin{aligned}
& \min _{W, S, P}\left(\left\|W^{T} X-W^{T} X S\right\|^{2}+\alpha\|S\|_{1}\right) \\
& +\beta \sum_{i, j}^{n}\left(\left\|W^{T} x_{i}-W^{T} x_{j}\right\|^{2} P_{i j}+\mu P_{i j}^{2}\right) \\
& +\gamma\|W\|_{21}
\end{aligned}
$$

Subject to, $S_{i i}=0, P 1_{n}=1_{n}, P \geq 0, W^{T} X X^{T} W=I$.

Where $\beta, \alpha, \gamma$, and $\mu$ are regularization parameters used to balance the adjustment error of global and local structure learning.

$X$ : considered as input Feature set $X \in R^{d \times n}, x_{i}=$ is the data sample. For each data sample $x_{i}$, entire set of the data points $\left\{x_{j}\right\}^{n} j=1$ are considered as the neighborhood of $x_{i}=$ with probability $P(i, j)$.

$S$ : Weight matrix of the data matrix.

$W$ : Feature selection and transformation matrix.

\subsection{Classification: Support vector machine(SVM) multiclass}

The SVM is used for binary classification. The set of examples and their corresponding labels is called learning set. An effective learning machine learns features of the training set and minimizes classification errors based on the set itself. $D=\left\{\left(x_{1}, y_{1}\right), . .,\left(x_{n}, y_{n}\right)\right\}$ represents the training set, with $x_{i} \in R^{m}$ and $y_{i}= \pm 1$. The objective of SVM is to maximize the margin between two classes by distinguishing them by a hyperplane. The optimal hyperplane requires the determination of the Euclidean distance between the hyperplane and the closest training of the two classes [27, 28]. The optimal hyperplane can also be solved by calculating the following classification function (17);

$f(x)=\sum_{i=1}^{l} \alpha_{i} y_{i} x^{T} x_{i}+b$

Where $y_{i}$ is the class label of support vector $x_{i}$. 
$x^{T}$ is a test tuple

$\alpha_{i}$ is a Lagrangian multiplier

$b$ is a numeric parameter

$l$ is the number of support vectors

The support vector machine (SVM) algorithm is one of the lazy learning techniques used for multiclass as well as is a relatively recent design learning model. The SVM multiclass case can be done in four different ways, which depends on the size of the set data: Directed Acyclic Graph (DAG), Binary Tree (BT), One-Against-One (OAO), and One-Against-All (OAA) classifiers.

In this study, one-against-one (OAO) of SVM multiclass using the radial basis function kernel (RBF) is chosen to discriminate between pathological cases. This method of multiclass is much faster to train and seems preferable for problems with a very large number of classes.

\subsection{Performances evaluation}

The performance of the classifier is estimated by calculating the Sensitivity $\left(S_{e}\right)$, the Specificity $\left(S_{p}\right)$, and the Accuracy (Acc). The sensitivity defines the true positive rate (18);

$S_{e}=\frac{T P}{T P+F N}$

The specificity defines the true negative rate as given in (19);

$S_{p}=\frac{T N}{F P+T N}$

The accuracy is defined as the ratio of correct predictions over the total number of predictions (20);

$A c c=\frac{T P+T N}{T P+T N+F P+F N}$

TP True positive: correctly classified as positive.

FP False positive: falsely classified as positive.

TN True negative: correctly classified as negative.

FN False negative: falsely classified as negative.

\subsection{Client-server architecture}

The communication mechanism in the client-server application allows exchanging data between them via the Internet using secured TCP/IP protocol (Transport Layer Security). So, the client-server architecture is developed around the use of socket and Threading. All communication between client and server is processed via sockets, which can be reliable. These sockets can be used in connected mode (TCP) or non-connected mode (UDP). The following steps describe the Communication process with the sockets in connected mode (see figure 3(a)).

As a first step, the creation of a socket is done using socket () function. Once the socket has been created, it has to be linked to a communication point defined by an address and a port, this is the role of the bind() function. In connected mode, the listen() function is used to put the socket in passive mode (listening to messages). In case of an incoming message, the connection can be accepted with the accept() function. When the connection has been accepted, the server receives the data using the $\operatorname{recv}()$ function. The end of the connection is done with the close() function.

In this study, the server can receive multiple client requests at the same time and receive each client request in parallel so that server won't be kept on hold [29]. The server uses the instances of a client object and individual threads to listen to the data that are being sent by each client while establishing new connections with the server. The main thread of the server creates a thread and forwards the client's request to this thread with its ID. The thread will start processing with the client request, generates the report, and send it back to the client. The figure 3(b) represents the process of the Threading function.

\section{Results}

In this study, we have developed a client-server application implemented as a telemedical platform for real-time remote monitoring for cardiac arrhythmia disease diagnosis. For the client block (hardware part), the electronic circuit is formed by an analog shaping and a data acquisition card (see figure 1). For the data acquisition card, we use the Raspberry Pi zero because it is more compact and suitable for narrower projects. It is characterized by its small dimensions, by its low energy consumption, and a very affordable price, in comparison with other types of Raspberry. The element parameters of the ECG sensor, the analog circuit, the communication system, and the server are listed in Table 2. ECGdata will be transmitted over an Internet network (TCP/IP) between the client and the server station using a Transport Layer Security protocol (TLS) that ensures the security of the transmitted data (see figure 7).

Concerning the server block (software part) (see figure 1), we developed a Graphical User Interface (GUI) to record the data acquisition task. The recorder ECG signal is then analyzed: we first estimate the HRV times series, then an SPWVD is applied on this HRV signal to extract features of interest. Finally, the learned SVM is applied to classify and label the analyzed ECG as AF and SVT, NSR and CHF. 


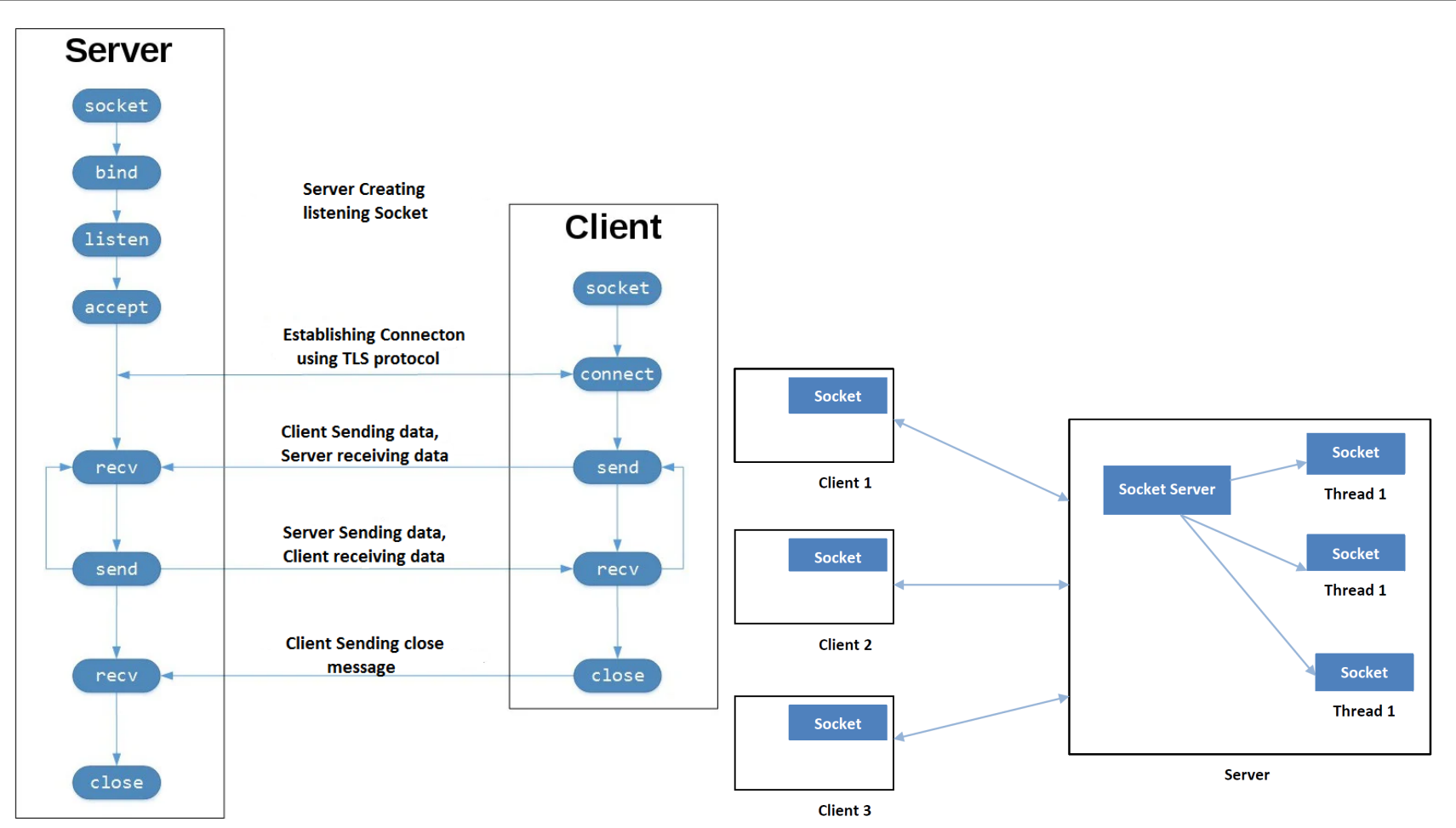

(a) State diagram for server and client model.

(b) Socket Server with Multiple Clients: implementing a Multithreaded Python Server.

Fig. 3: Client-server architecture using socket and Threading.

Table 2: Element Parameters of the cardiac arrhythmia monitoring system.

\begin{tabular}{lll}
\hline & Parameters & Vlaues \\
& & \\
ECG sensor & Type & AD8232 \\
& Supply Voltage & $2.0 \mathrm{~V}-3.5 \mathrm{~V}$ \\
Analog circuit & Type & Amplification, Filtering \\
& Type & MCP3008 \\
ADC & Interface & SPI, Serial \\
& Supply Voltage & $2.7 \mathrm{~V}-5.5 \mathrm{~V}$ \\
& Output Voltage & $4.1 \mathrm{~V}$ \\
& Number of Bits & 10 \\
& & \\
Raspberry Pi & Type & Raspberry pi zero \\
& Power voltage & $1.24 \mathrm{~A}-5 \mathrm{~V}$ \\
& Core & 32 bit ARM1176JZF-S \\
& GPU & single-core \\
& CPU clock & $1 \mathrm{GHz}$ \\
& Memory & $512 \mathrm{MB}$ \\
Communication & Wireless transmission & $3 \mathrm{G} / 4 \mathrm{G}$ \\
system & protocols & \\
& communications security & TLS Protocol \\
Server & CPU & Intel(R) Core(TM) i53230M \\
& Operation system & Windows 10, 64 bit \\
& &
\end{tabular}

3.1 Real—time QRS complex-based detection of the HRV signal

In this study, we used the online implementation of the PanTomkins algorithm to detect the HRV from real-time acquired ECG signal (see figure 4(a)). The HRV signal illustrated in figure 4(c) is detected by localizing R-peaks in relation to cardiac cycles within real-time ECG signal as represented in figure 4(b). The amplitude within HRV signals refers to the consecutive duration between $\mathrm{R}$-peaks within ECG signals. Therefore, HRV signals will be amplitudecentered around the cardiac cycle mean value. We performed detection of the R-peaks within the ECG through regular real-time localization of the consecutive maxima which we used as cardiac cycles.

\subsection{Identification of features of interest and SVM parameters learning}

The Physionet database is used to help us to choose the best features and to learn the SVM parameters. More precisely, 228 ECG signals divided into four classes, namely SVT, NSR, AF, and CHF. (See Data set section) are exploited. Figure 5 shows an example the SPWVD calculated for the four different classes. Clearly, the shape and the TF distribu- 


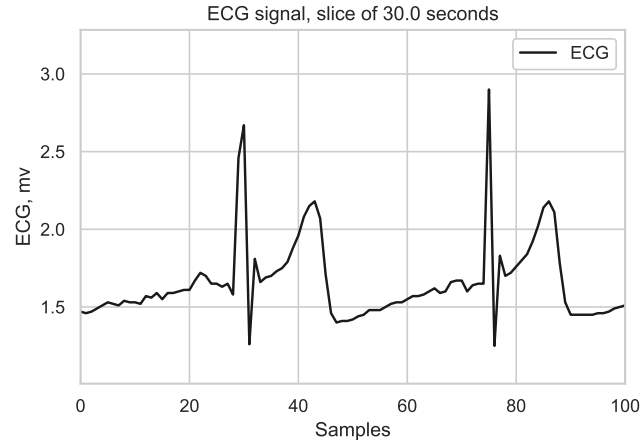

(a) Acquired ECG signal from data acquisition system

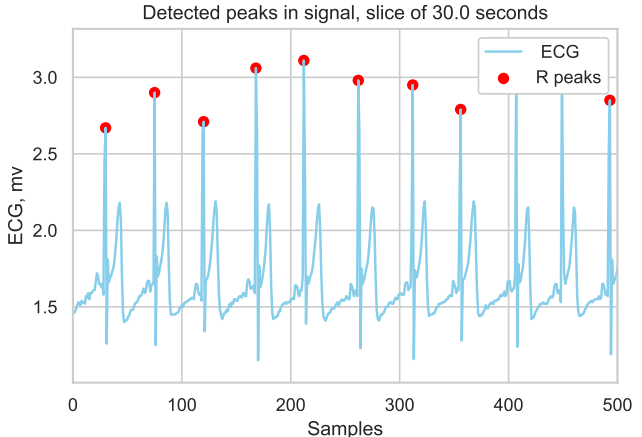

(b) Cardiac cycles detected from an acquired ECG signal

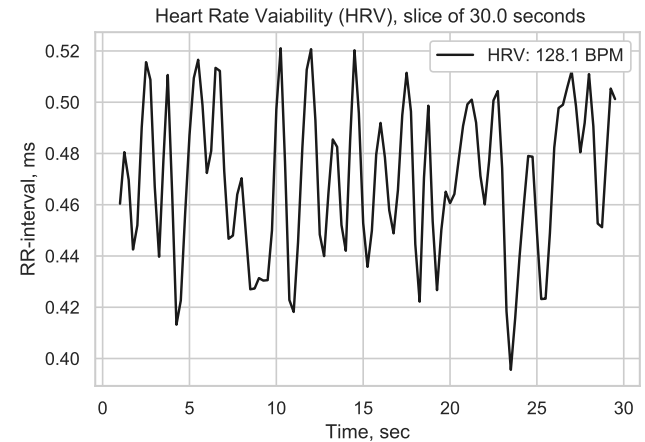

(c) AC component of the detected HRV signal from the ECG signal of Figure 4(a)

Fig. 4: ECG and HRV signals.

tion of the amplitude of the spectra are different between the four classes, which confirm that the analysis of HRV signals in TF domain is a good choice instead of the frequency domain. In addition, we can also remark that SPWVD provides a good resolution in both time and frequency domains, with less cross-terms.

Figure 6 depicts the mean and standard deviation values of eight features (see Feature extraction section ), calculated from the TF distributions of HRV signals, for NSR, CHF, $\mathrm{AF}$, and SVT. We can observe that mean and standard deviation values of each feature are clearly different between the different classes. However, the manual selection of the best set of features is not straightforward and the testing all combinations will be very times consuming. The automatic selection of the best features using FSASL and MI methods is reported in Table 3. It is interesting to see that the MI method is not able to select a set of features between the eight ones, whereas the FSASL algorithm select just three valuable feature, namely Coef.variation, Skewness and Shannon Entropy. Regarding the classification rate, we can observe that our approach combining SPWVD and SVM, seems to be very effective in discriminating between SVT, AF, CHF, and NSR classes (see the results of Table 3). In addition, the best classification rate is yield using the FSASL features selection algorithm: i) $\mathrm{Se}=95.65 \%, \mathrm{Sp}=98.55 \%$ and $\mathrm{Acc}=97.82 \%$, and ii) $\mathrm{Se}=91.30 \%, \mathrm{Sp}=95.65 \%$ and Acc $=94.20 \%$ (using MI). Table 4 presents, in detail, the classification rate of AF and SVT among CHF and NSR, using the features selected by the FSASL. The global proposed procedure seems to be very efficient whatever the classified pathology. Note that, all the previous results are obtained by randomly dividing the database into a training set $(90 \%$ of ECG) and a testing set (10\% ECG) using a bootstrap strategy.

\section{Discussion}

In this study, the server and the client could initiate a communication via the Graphical user interface (GUI) by using one IP address, the following procedure explains how the client connects to the server in different cases:

- Run the client on the same computer as the server, it uses the IP address as the host name.

- Run the client in the same network as the server's (local network), it uses the local server IP address in the client interface.

- The port can be any 16-bit number, but must be forwarded to the router where the client is located, if the 

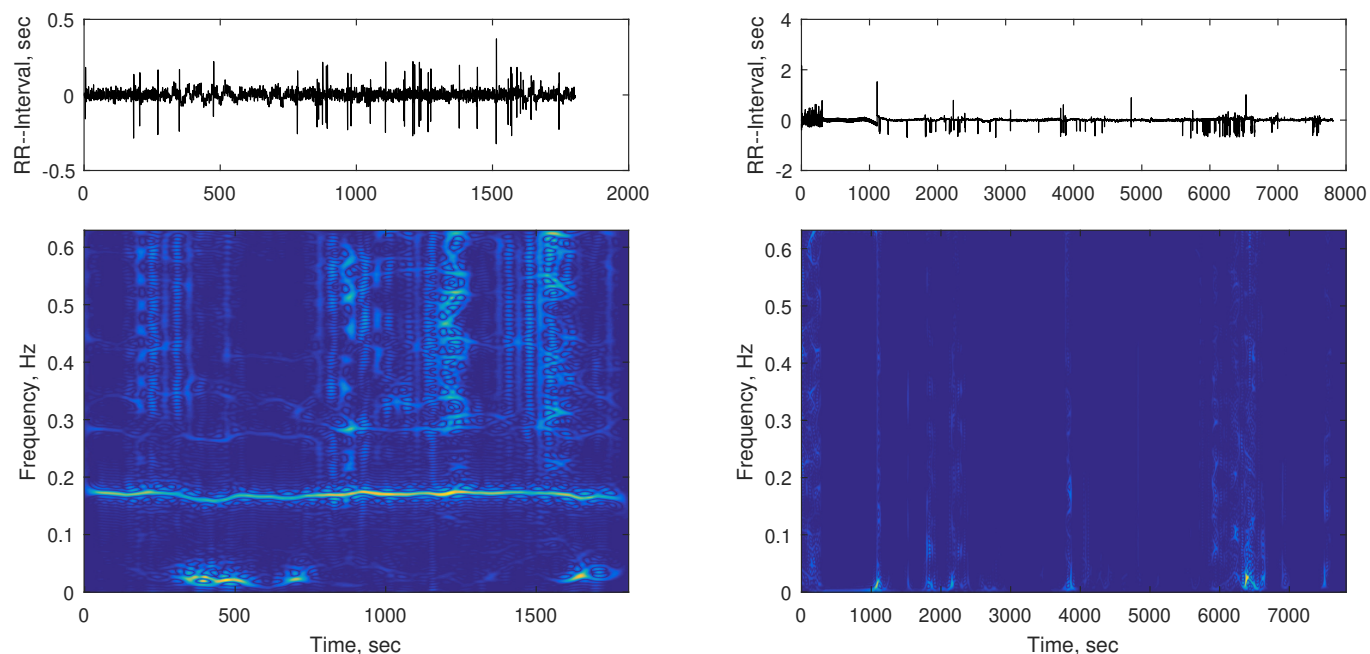

(a) HRV times series and SPWVD of normal sinus rhythm (b) HRV times series and SPWVD of atrial fibrillation (AF) (NSR)
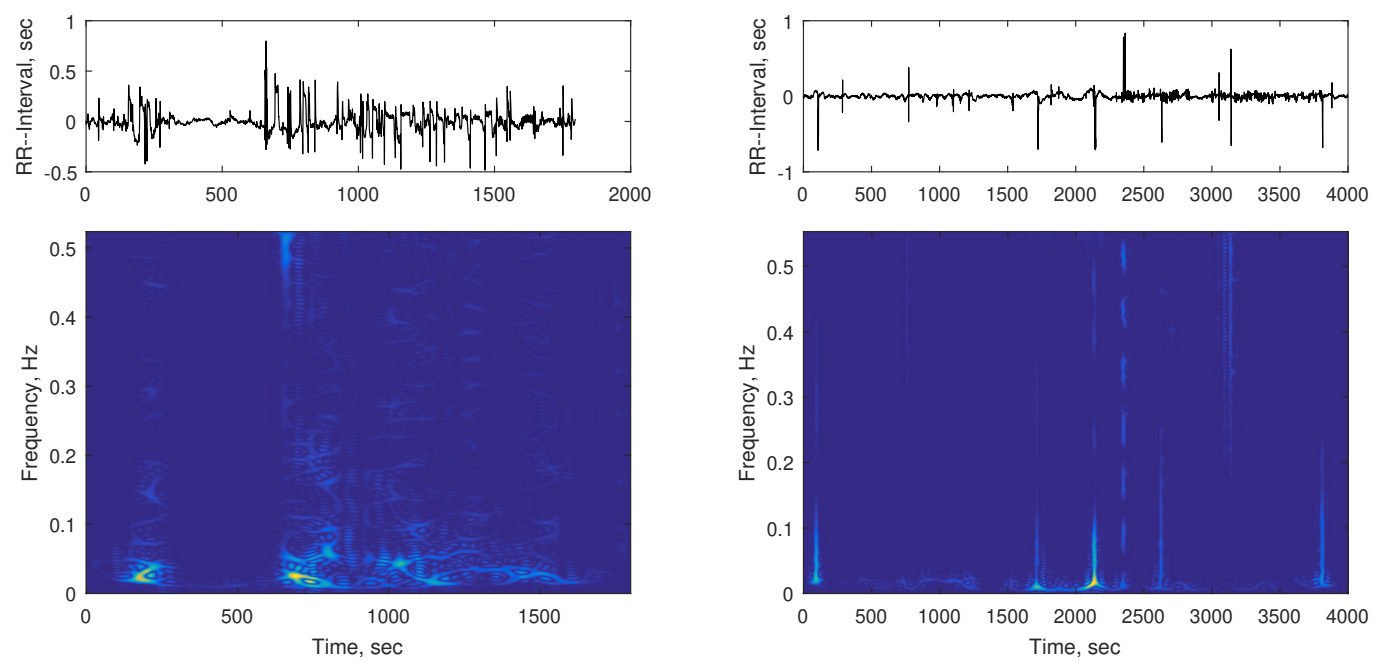

(c) HRV times series and SPWVD of supraventricular tachy- (d) HRV times series and SPWVD of congestive heart failure cardia (SVT) (CHF)

Fig. 5: Time-frequency analysis of the detected HRV signal for different classes.

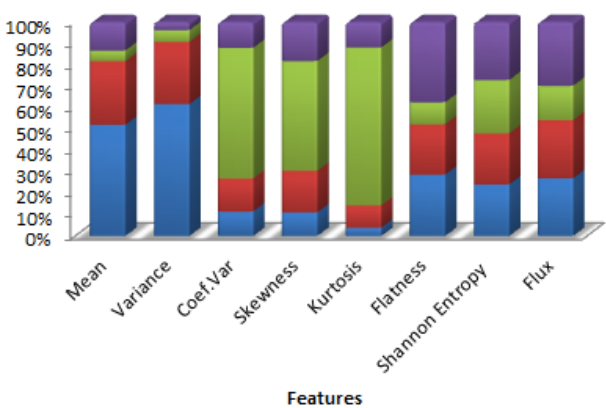

(a) Mean value

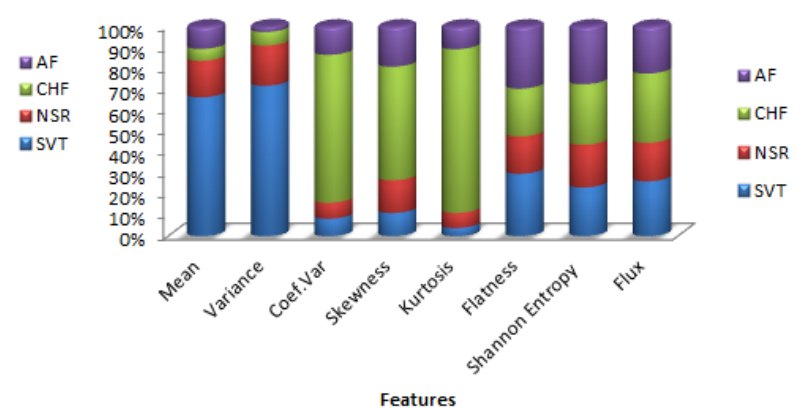

(b) Standard deviation value

Fig. 6: Statistical result of various Features extraction from TF-representation of HRV signal for different classes. 
Table 3: Result of classification using MI and FSASL as feature selection methods.

\begin{tabular}{cccccc}
\hline \multirow{2}{*}{ FSM } & \multicolumn{5}{c}{ Metrics performance } \\
\cline { 2 - 6 } & NF & FS & Sensitivity (\%) & Specificity (\%) & Accuracy (\%) \\
\hline MI & 08 & $\begin{array}{c}\text { Mean, Variance, Coef.variation, Kurtosis } \\
\text { Shannon Entropy, Skewness, Flux, Flatness }\end{array}$ & 91.30 & 95.65 & 94.20 \\
FSASL & $\mathbf{0 3}$ & Coef.variation, Skewness, Shannon Entropy & $\mathbf{9 5 . 6 5}$ & $\mathbf{9 8 . 5 5}$ & $\mathbf{9 7 . 8 2}$ \\
\hline
\end{tabular}

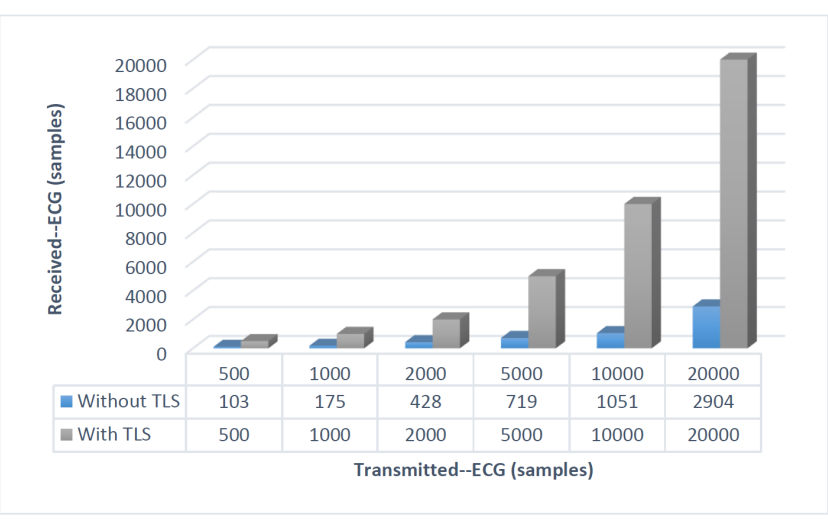

Fig. 7: Transmission process with and without using the security protocol (TLS).

Table 4: Classification result of cardiac arrythmia among NSR and CHF using FSASL.

\begin{tabular}{lccc}
\hline \multirow{2}{*}{ Classes } & \multicolumn{3}{c}{ Metrics performance } \\
\cline { 2 - 4 } & $\mathrm{Se}(\%)$ & $\mathrm{Sp}(\%)$ & $\mathrm{Acc}(\%)$ \\
\hline Atrial Fibrillation (AF) & 100 & 100 & 100 \\
Supraventricular Tachycardia (SVT) & 83.33 & 100 & 95.65 \\
Normal Sinus Rhythm (NSR) & 100 & 95.23 & 95.65 \\
Congestive Heart Failure (CHF) & 100 & 100 & 100 \\
Overall Accuracy & 95.65 & 98.55 & 97.82 \\
\hline
\end{tabular}

server and client are located in a different network, the client uses the IP address (internet IP) of the network under which the server is running.

The Tele-vigilance station can communicate with the various remote stations through a secure communication system to exchange the data and the processing results of the data collected. Transport Layer Security has been widely recognized as one of the most widely used cryptography protocols to protect and secure data transmitted between client and server. For this, the hacker retrieves only the encrypted data. TLS protocol uses a certificate and key to establish encryption between server and client. In this paper, We have used the TLS protocol (version 1.2) to secure the exchange of data transmitting over the internet network. TLS version 1.2 has more than 300 cipher suites registered on the Internet Assigned Numbers Authority (IANA). Figure 10 describes the transmission of ECG-data using TLS protocol and without TLS protocol. As results, we found that there is a loss of information between the transmitter and receiver during transmission of ECG-data without using TLS protocol. On the other hand, we acquired the same data in the receiver as the transmitter when we used the TLS protocol. This result confirms the importance of using the data security protocol during transmission of ECG-data over wireless interface.

Recently, many studies have been developed and proposed a monitoring system to acquire, encrypting and transmitting over the internet network to the cloud server to store, processing, classification, and diagnosis. However, we compared our system with the others systems proposed through the use of security protocols during the transmission of collecting data to provide better and safer care for patients. Francesca Stradolini et al. [30] developed a first architecture for continuous monitoring of anesthesia. But, no cloud integration was provided in this architecture. However, in [31] ,they present the development of an IoT cloud-based solution dedicated to anesthesia monitoring during surgeries. An IoT platform for the prediction of cardiovascular disease using an IoT-enabled ECG telemetry system has been studied in [13-16]. Khalid abusalim et al. [14] describes a cloudintegrated IoT monitoring framework, where ECG-data are watermarked before being sent to the cloud for secure. In [13], a secure monitoring platform has been developed to share health information between the patient and his doctors or healthcare professionals securely and confidentially. A new wearable ECG system has been proposed to reliably send the ECG signal to the cloud server over MultiArts with lightweight security was proposed in [16]. The cardiac arrhythmia diseases such as ventricular tachycardia, atrial fbrillation, supraventricular tachycardia, and atrial futter was studied in [15]. For this purpose, an IoT-enabled ECG monitoring system for cardiac arrhythmia disease di- 
Table 5: Comparison between some IoT solutions in health monitoring.

\begin{tabular}{|c|c|c|c|c|c|}
\hline Works & Medical Application & Architecture & Cloud & Security Protocol & $\begin{array}{c}\text { Hospital (HS) or } \\
\text { Home (HM) } \\
\text { Monitoring }\end{array}$ \\
\hline [13] & $\begin{array}{l}\text { Elderly patients health } \\
\text { monitoring }\end{array}$ & $\begin{array}{l}\text { Cloud storage provider, } \\
\text { ECG sensor, mobile } \\
\text { device app }\end{array}$ & $\sqrt{ }$ & $\sqrt{ }$ & HM \\
\hline [14] & $\begin{array}{l}\text { ECG health } \\
\text { monitoring }\end{array}$ & $\begin{array}{l}\text { Cloud system, ECG } \\
\text { sensor, mobile device } \\
\text { app, desktop software }\end{array}$ & $\sqrt{ }$ & $\sqrt{ }$ & $\mathrm{HM}$ \\
\hline [15] & $\begin{array}{l}\text { IoT-based cardiac } \\
\text { arrhythmia diagnosis }\end{array}$ & $\begin{array}{l}\text { ECG sensor, Cloud system, } \\
\text { Statistical Features,HRV } \\
\text { SVM, mobile device app }\end{array}$ & $\sqrt{ }$ & $\times$ & HM \\
\hline$[16]$ & $\begin{array}{l}\text { IoT-based monitoring, } \\
\text { collecting ECG-Data }\end{array}$ & $\begin{array}{l}\text { ECG sensor, could server, } \\
\text { for storing and further } \\
\text { processing }\end{array}$ & $\sqrt{ }$ & $\sqrt{ }$ & $\mathrm{HM}$ \\
\hline [30] & Anesthesia monitoirng & $\begin{array}{l}\text { Therapeutic Drug Monitoring } \\
\text { system, mobile device app, } \\
\text { smartwatch }\end{array}$ & $\times$ & $\times$ & HS \\
\hline [31] & Anesthesia monitoring & $\begin{array}{l}\text { Pryv middleware cloud } \\
\text { cloud,mobile device app, } \\
\text { smartwatch, WebApp }\end{array}$ & $\sqrt{ }$ & $\times$ & HS \\
\hline Our work & $\begin{array}{l}\text { Telemedical platform, } \\
\text { cardiac arrhythmia } \\
\text { classification }\end{array}$ & $\begin{array}{l}\text { ECG sensor, could server } \\
\text { TLS, HRV, TF-Analysis } \\
\text { TF-Features, Features selection } \\
\text { SVM, client-server app }\end{array}$ & $\sqrt{ }$ & $\sqrt{ }$ & HM \& HS \\
\hline
\end{tabular}

agnosis was developed. However, [15], [30], and [31] developed a novel m-Health system that can be used to monitor patients wirelessly. Nevertheless, no security protocol has been provided in these studies

In our study, we designed a new low-energy consumption, and a very affordable price system capable of performing continuous recording of HRV times series for easy monitoring of the heart rhythm for the detection of arrhythmia. We used an electronic circuit formed by an analog shaping part and a data acquisition system. As a software part, we developed a Graphical User Interface (GUI) within a python environment to establish the connection between the client and the server through the TCP/IP secured by the Transport Layer Security, to acquire, transmit, monitor, process and classify the hearth rhythm. We developed a new approach that combines SPWVD and SVM, for analyzing and classifying HRV signals. More precisely, three features of interest were extracted from the TF-representation of HRV signal: the coefficient of variation, the skewness, and the Shannon entropy. The obtained results show clearly that the proposed system is very efficient to differentiate between cardiac arrhythmia as AF and SVT among CHF and NSR cases. The result of classification can be sent to the medical professional for analysis and diagnosis.

\subsection{Client-server application}

The graphical user interface (client-server) is dedicated to remote monitoring of cardiac arrhythmia, display, archiving, digital signal processing, classification, and transmission of ECG-data to a remote station under a secured TCP/IP protocol.

Client application is a graphical interface implemented with python environment connected with a data acquisition system to acquire the ECG signal for transmitting over internet network to the remote station (server). The client application is represented in figure 8(a) and involves:

- Connection establishment window: relating to the communication phase between the server and the client (establish and open the connection through an internet network using the server's IP address). 


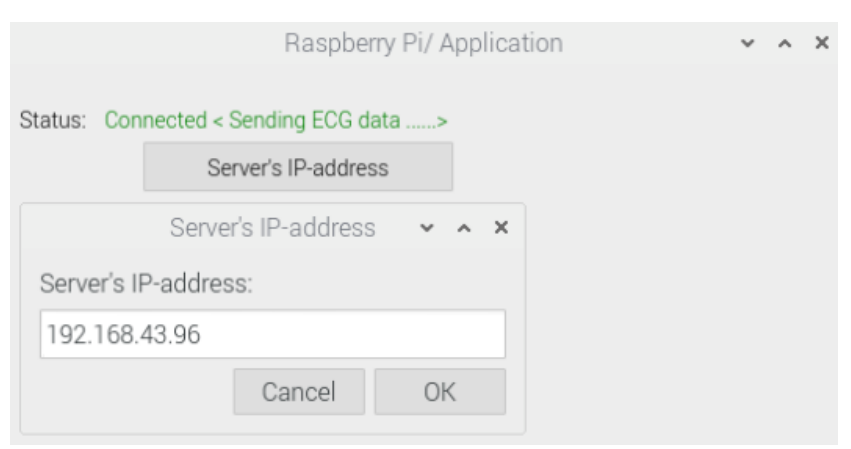

(a) Client Application: send ECG-signal from the Raspberry PI to the Server.

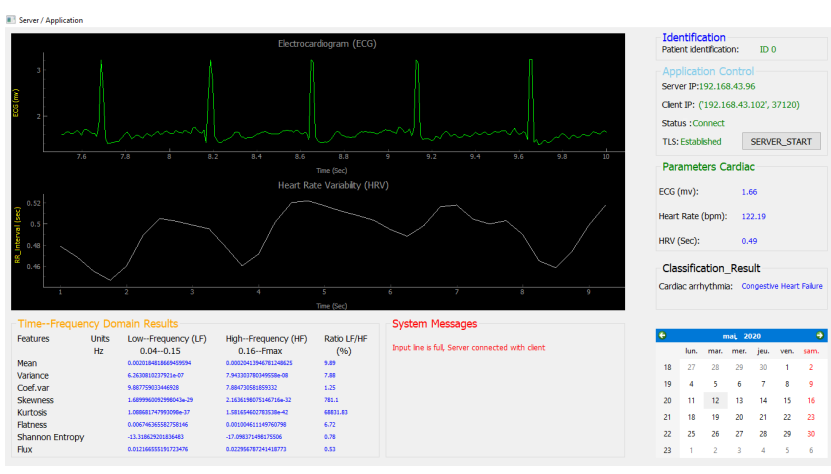

(b) Tele-monitoring and classification of cardiac arrhythmia.

Fig. 8: Telemedical transport layer security based platform for cardiac arrhythmia classification .

Server application is implemented using the python environment, consists of visualization, archiving, processing of HRV signal, and classification of received ECG signal. The server application is illustrated in figure $8(\mathrm{~b})$ and involves:

- Control window for viewing and recording the signal: allows to view and record the ECG signal and its corresponding HRV in real-time.

- Consultation window: allows measuring some parameters related to the heart rate such as heart rate (bpm), average HRV value (sec), and ECG signal values (mv).

- Connection establishment window: this phase establishes the communication between the server and the client under the secured TCP/IP protocol.

- Digital processing window: is devoted to analyze the HRV times series, by calculating there TF distribution and extraction of the three best features.

- Classification window: the learned SVM is used to classify the new HRV signal.

- Message window: used to display and indicate messages corresponding to the application.

\section{Acknowledgments}

The authors would like to thank the Directorate General of Scientific Research and Technological Development (Direction Générale de la Recherche Scientifique et du Développement Technologique, DGRSDT, URL:www.dgrsdt.dz, Algeria) for the financial assistance towards this research.

\section{Data Availability}

The data used in the current study are available in the PhysioNet repository, managed by the MIT Laboratory for Computational Physiology. For more information about the data used in this study, visit https://physionet.org.

\section{Conflicts of Interest}

The authors declare that there is no conflict of interest.

\section{References}

1. Arife Iimen Atalar, Feray Karaali Savrun, and Seher Naz Yeni. Autonomic dysfunction during the interictal period: an electrophysiologic study. Neurological sciences and neurophysiology, 36(1):9-15, 2019.

2. Tristan W Dorey, Motahareh Moghtadaei, and Robert A Rose. Altered heart rate variability in angiotensin ii mediated hypertension is associated with impaired autonomic nervous system signaling and intrinsic sinoatrial node dysfunction. Heart Rhythm, 2020.

3. Rachele Anderson, Peter Jönsson, and Maria Sandsten. Stochastic modeling and optimal time-frequency estimation of task-related hrv. Applied Sciences, 9(23): 5154, 2019.

4. Rohan Joshi, Deedee Kommers, Chengcheng Guo, JanWillem Bikker, Loe Feijs, Carola van Pul, and Peter Andriessen. statistical modeling of heart rate variability to unravel the factors affecting autonomic regulation in preterm infants. Scientific reports, 9(1):1-9, 2019.

5. Maddalena Ardissino, Nicoletta Nicolaou, and Marcela Vizcaychipi. Non-invasive real-time autonomic function characterization during surgery via continuous poincaré quantification of heart rate variability. Journal of clinical monitoring and computing, 33(4):627-635, 2019.

6. Du-Yan Geng, Jie Zhao, Chen-Xu Wang, and Qi Ning. A decision support system for automatic sleep staging from hrv using wavelet packet decomposition and energy features. Biomedical Signal Processing and Control, 56:101722, 2020. 
7. RS Singh, BS Saini, and RK Sunkaria. Power spectral study of heart rate variability time series by the adaptive modified continuous morlet wavelet transform. Research \& Reviews: Journal of Medical Science and Technology, 6(2):5-20, 2019.

8. MG Poddar, Anjali C Birajdar, Jitendra Virmani, et al. Automated classification of hypertension and coronary artery disease patients by pnn, knn, and svm classifiers using hrv analysis. In Machine Learning in Bio-Signal Analysis and Diagnostic Imaging, pages 99-125. Elsevier, 2019.

9. Mai Kobayashi, Guanghao Sun, Toshikazu Shinba, Takemi Matsui, and Tetsuo Kirimoto. Development of a mental disorder screening system using support vector machine for classification of heart rate variability measured from single-lead electrocardiography. In 2019 IEEE Sensors Applications Symposium (SAS), pages 16. IEEE, 2019.

10. Yi-Chun Chen, Chun-Chieh Hsiao, Wen-Dian Zheng, Ren-Guey Lee, and Robert Lin. Artificial neural networks-based classification of emotions using wristband heart rate monitor data. Medicine, 98(33), 2019.

11. Laura Verde and Giuseppe De Pietro. A neural network approach to classify carotid disorders from heart rate variability analysis. Computers in biology and medicine, 109:226-234, 2019.

12. Jiayuan He and Ning Jiang. Optimizing probability threshold of convolution neural network to improve hrvbased acute stress detection performance. In $201941 \mathrm{st}$ Annual International Conference of the IEEE Engineering in Medicine and Biology Society (EMBC), pages 5318-5321. IEEE, 2019.

13. Danan Thilakanathan, Shiping Chen, Surya Nepal, Rafael Calvo, and Leila Alem. A platform for secure monitoring and sharing of generic health data in the Cloud. Future Generation Computer Systems, 35:102113, jun 2014. doi: 10.1016/j.future.2013.09.011.

14. M. Shamim Hossain and Ghulam Muhammad. Cloudassisted Industrial Internet of Things (IIoT) - Enabled framework for health monitoring. Computer Networks, 101:192-202, jun 2016. ISSN 13891286. doi: 10.1016/j.comnet.2016.01.009.

15. R. Lakshmi Devi and V. Kalaivani. Machine learning and IoT-based cardiac arrhythmia diagnosis using statistical and dynamic features of ECG. Journal of Supercomputing, 76(9):6533-6544, sep 2020. doi: 10.1007/s11227-019-02873-y.

16. Khalid Abualsaud, Muhammad E.H. Chowdhury, Abdurrazzak Gehani, Elias Yaacoub, Tamer Khattab, and Jamal Hammad. A New Wearable ECG Monitor Evaluation and Experimental Analysis: Proof of Concept. In 2020 International Wireless Communications and Mobile Computing, IWCMC 2020, pages 1885-1890. In- stitute of Electrical and Electronics Engineers Inc., jun 2020. doi: 10.1109/IWCMC48107.2020.9148191.

17. Ary L Goldberger, Luis AN Amaral, Leon Glass, Jeffrey M Hausdorff, Plamen Ch Ivanov, Roger G Mark, Joseph E Mietus, George B Moody, Chung-Kang Peng, and $\mathrm{H}$ Eugene Stanley. Physiobank, physiotoolkit, and physionet: components of a new research resource for complex physiologic signals. circulation, 101(23): e215-e220, 2000.

18. Jiapu Pan and Willis J Tompkins. A real-time qrs detection algorithm. IEEE transactions on biomedical engineering, (3):230-236, 1985.

19. Boualem Boashash. Time-frequency signal analysis and processing: a comprehensive reference. Academic Press, 2015.

20. Boualem Boashash and Samir Ouelha. An Improved Design of High-Resolution Quadratic Time-Frequency Distributions for the Analysis of Nonstationary Multicomponent Signals Using Directional Compact Kernels. IEEE Transactions on Signal Processing, 65(10):2701-2713, may 2017. doi: 10.1109/TSP.2017.2669899.

21. Boualem Boashash, Ghasem Azemi, and Nabeel Ali Khan. Principles of time-frequency feature extraction for change detection in non-stationary signals: Applications to newborn EEG abnormality detection. Pattern Recognition, 48(3):616-627, mar 2015. doi: 10.1016/J.PATCOG.2014.08.016.

22. Johan Löfhede, Magnus Thordstein, Nils Löfgren, Anders Flisberg, Manuel Rosa-Zurera, Ingemar Kjellmer, and Kaj Lindecrantz. Automatic classification of background EEG activity in healthy and sick neonates. Journal of Neural Engineering, 7(1):016007, feb 2010. doi: 10.1088/1741-2560/7/1/016007.

23. Ron Kohavi and George H. John. Wrappers for feature subset selection. Artificial Intelligence, 97(1-2):273324, dec 1997. doi: 10.1016/S0004-3702(97)00043-X.

24. Isabelle Guyon and André Elisseeff. An Introduction to Variable and Feature Selection. Journal of Machine Learning Research, 3(Mar):1157-1182, 2003.

25. Hanchuan Peng, Fuhui Long, and Chris Ding. Feature selection based on mutual information criteria of max-dependency, max-relevance, and min-redundancy. IEEE Transactions on pattern analysis and machine intelligence, 27(8):1226-1238, 2005.

26. Liang Du and Yi Dong Shen. Unsupervised feature selection with adaptive structure learning. In Proceedings of the ACM SIGKDD International Conference on Knowledge Discovery and Data Mining, volume 2015August, pages 209-218. Association for Computing Machinery, aug 2015. doi: 10.1145/2783258.2783345.

27. Shigeo Abe. Support vector machines for pattern classification. Springer, 2005. 
28. Jin-Hyuk Hong and Sung-Bae Cho. A probabilistic multi-class strategy of one-vs.-rest support vector machines for cancer classification. Neurocomputing, 71(16-18):3275-3281, oct 2008. doi: 10.1016/j.neucom.2008.04.033.

29. Jintae Kim, Youngmin Kim, Natnael S. Zewge, and Jong Hwan Kim. A Robust Client-Server Architecture for Map Information Processing and Transmission for Distributed Visual SLAM. In 2019 7th International Conference on Robot Intelligence Technology and Applications, RiTA 2019, pages 99-105. Institute of Electrical and Electronics Engineers Inc., nov 2019. doi: 10.1109/RITAPP.2019.8932869.

30. Francesca Stradolini, Eleonora Lavalle, Giovanni De Micheli, Paolo Motto Ros, Danilo Demarchi, and
Sandro Carrara. Paradigm-shifting players for IoT: Smart-watches for intensive care monitoring. In Lecture Notes of the Institute for Computer Sciences, Social-Informatics and Telecommunications Engineering, LNICST, volume 192, pages 71-78. Springer Verlag, 2017. doi: 10.1007/978-3-319-58877-3_9.

31. Francesca Stradolini, Nadia Tamburrano, Thiebaud Modoux, Abuduwaili Tuoheti, Danilo Demarchi, and Sandro Carrara. IoT for Telemedicine Practices enabled by an Android ${ }^{\mathrm{TM}}$ Application with Cloud System Integration. In Proceedings - IEEE International Symposium on Circuits and Systems. Institute of Electrical and Electronics Engineers Inc., apr 2018. doi: 10.1109/ISCAS.2018.8351871. 